\title{
A plasmon-based nanoruler to probe the mechanical properties of synthetic and biogenic nanosized lipid vesicles
}

Lucrezia Caselli, ${ }^{\mathrm{a}, \mathrm{b}}$, Andrea Ridolfi ${ }^{\mathrm{a}, \mathrm{b}, \mathrm{c}}$, Jacopo Cardellini ${ }^{\mathrm{a}, \mathrm{b}}$, Lewis Sharpnack ${ }^{\mathrm{d}}$, Lucia Paolini ${ }^{\mathrm{b}, \mathrm{e}}$, Marco Brucale $^{b, c}$, Francesco Valle ${ }^{b, c}$, Costanza Montis*a,b, Paolo Bergese ${ }^{b, e, f}$ and Debora Berti*a,b

${ }^{a}$ Department of chemistry, University of Florence, Via della Lastruccia 3, Sesto Fiorentino, 50019 Florence, Italy.

${ }^{b}$ Consorzio Sistemi a Grande Interfase, Department of Chemistry, University of Florence, Sesto Fiorentino, Italy 'Consiglio Nazionale delle Ricerche, Istituto per lo Studio dei Materiali Nanostrutturati (CNRISMN), via Gobetti 101, 40129 Bologna (Italy).

${ }^{d}$ ESRF - The European Synchrotron, 38043 Grenoble, France

${ }^{e}$ Department of Molecular and Translational Medicine, University of Brescia, Brescia, Italy

${ }^{f}$ Consorzio Interuniversitario Nazionale per la Scienza e la Tecnologia dei Materiali, Florence, Italy

Membrane-delimited compartments, as lipid vesicles, are ubiquitous in natural and synthetic systems. The mechanical properties of such vesicles are crucial for several physical, chemical, and biological processes. However, their accurate determination is still challenging and requires sophisticated instruments and data analysis. Here we report the first evidence that the surface plasmon resonance (SPR) of citrated gold nanoparticles (AuNPs) adsorbed on synthetic vesicles is finely sensitive to the vesicles' mechanical properties. We leverage this finding to demonstrate that the spectrophotometric tracking of the SPR provides quantitative access to the stiffness of vesicles of synthetic and natural origin, such as extracellular vesicles (EVs). This plasmon-based "stiffness nanoruler" paves the way for developing a facile, cost-effective, and high-throughput method to assay the mechanical properties of vesicles of nanometric size and unknown composition.

\section{Introduction}

Membrane-delimited compartments (e.g., cells, organelles, enveloped viruses, biogenic vesicles, etc.) are among the basic units of living organisms and widespread structural motifs in bio-inspired nanomaterials, such as liposomes. Their mechanical properties, which regulate the response to external deformations, are crucial in a host of biologically-relevant interactions at the nanoscale ${ }^{1-5}$. The deformability of 
liposomes or polymeric vesicles for drug delivery affects their pharmacokinetics in the bloodstream and their internalization mechanisms ${ }^{6}$. The mechanical properties of cells and membrane bound-organelles are key in numerous biological processes (e.g. cell fusion, growth and differentiation, endo- and exocytosis, uptake of nanoparticles or viruses ${ }^{7-9}$, etc.) and in the onset of pathological cell conditions ${ }^{10-}$ 13. Recent reports have highlighted that the mechanical response of extracellular vesicles, (EVs, membrane-delimited nanoparticles secreted by all cell types and essential mediators of cell signalling ${ }^{14-}$ ${ }^{16}$ ) is a biomarker for malignant conditions of parental cells ${ }^{17,18}$. In addition, the nanomechanics of pathogens, including viruses with a lipid envelope (e.g. Moloney murine leukemia virus and HIV ${ }^{19}$ ) was recently connected to their infectivity ${ }^{20}$.

Although central in several research areas, the accurate assessment of the mechanical properties of synthetic or natural vesicles still poses a challenge ${ }^{21,22}$. Traditional methods provide insights into biologically-relevant descriptors of the mechanical response of the lipid membrane, such as the bilayer's bending rigidity ${ }^{23-27}$. However, these techniques are cost- and time-consuming, and often yield discrepant results, as pointed out in several reports ${ }^{21,22,28-30}$. More recently, techniques that actively probe the mechanical properties at a whole-vesicle level, rather than those of the lipid shell (e.g. Atomic Force Microscopy-based Force Spectroscopy (AFM-FS), optical tweezers, etc.) are gaining the central stage ${ }^{31}$. These methods allow determining the overall mechanical response of the vesicle to applied deformations, i.e., its "stiffness". The measured stiffness includes contributions both from the membrane shell and the enclosed volume, accounting for the mechanical properties of the internal pool, volume variations upon deformation, osmotic imbalance, etc. Unfortunately, the experimental methods proposed so far probe a single particle at a time and require sophisticated instruments or/and highly experienced users ${ }^{31}$.

Here, we propose AuNPs as nanoprobes of the stiffness of membranous nano-objects, with typical submicron sizes. This approach overcomes many limitations of the currently available methods, measurements can be performed with an UV-Vis spectrophotometer and limited data analysis is required. In the following, this communication will (i) explore how the stiffness of liposomes modulates the surface plasmon resonance (SPR) of AuNPs adsorbed on their membrane and (ii) propose this previously unnoticed relationship as the working principle of a new, accessible and robust spectrophotometric method to evaluate the stiffness of both synthetic and natural lipid vesicles of unknown composition.

The SPR of AuNPs is finely sensitive to the chemical environment and the interparticle distance, which underpins their application as nanoscale probes ${ }^{32}$. The coupling between the SPR of proximal AuNPs, which results from AuNPs close approach or aggregation, was exploited for the first time by El-Sayed and 
co-workers as a plasmon ruler ${ }^{33}$ and is nowadays used in a number of bioanalytical assays ${ }^{34,35}$. The CONAN (COlloidal NANoplasmonic) assay is a recent example, where the AuNPs SPR shift upon incubation with EVs is exploited to determine their purity and concentration ${ }^{36-38}$; in this latter case, the SPR shift arises from the spontaneous aggregation of AuNPs on the lipid membrane of vesicles (of both synthetic and natural origin, as EVs). This membrane-induced aggregation has been the focus of several recent investigations ${ }^{39-44}$. Specifically, the membrane-induced aggregation of AuNPs has been interpreted as onoff mechanism to date ${ }^{45,46}$, switchable by the physical state of the membrane: fluid-phase bilayers, characterized by free lipid diffusion and low rigidity, would promote aggregation, resulting in a marked change of AuNPs SPR profile. Conversely, the aggregation of AuNPs would be completely inhibited on tightly packed gel-phase membranes, characterized by a higher rigidity. Here we demonstrate instead that the SPR shift of AuNPs shows a functional dependence on the stiffness of synthetic vesicles, which allows defining a "stiffness nanoruler". In analogy with the plasmon nanoruler, introduced as distance-sensor ${ }^{33}$, this plasmon-based descriptor leverages the unique sensitivity of AuNPs SPR to determine the mechanical properties of lipid vesicles. As a proof-of-principle of applicability to complex natural systems, we tested the assay on EVs, whose stiffness is of prominent relevance in cellular adhesion and uptake ${ }^{47}$ and a characteristic that distinguish EVs deriving from malignant and nonmalignant cells ${ }^{17,18}$.

\section{Results and Discussion}

We prepared a library of unilamellar liposomes having a similar average diameter ( $100 \mathrm{~nm}$ ) and low polidispersity indexes (see Supporting Information for details on preparation and characterization) from a set of synthetic phosphatidylcholines (PC) differing for length and/or degree of unsaturation of the acyl chains (Figure 1a). The free-standing bilayers, either in the gel or fluid phase at room temperature (Figure 1a), display different rigidities ${ }^{48-51}$. Given their very similar size distributions and the absence of any osmotic imbalance between the lumen and the external medium, the rigidity of the lipid shells can be considered the sole responsible for the overall stiffnesses of the vesicles. 
a)

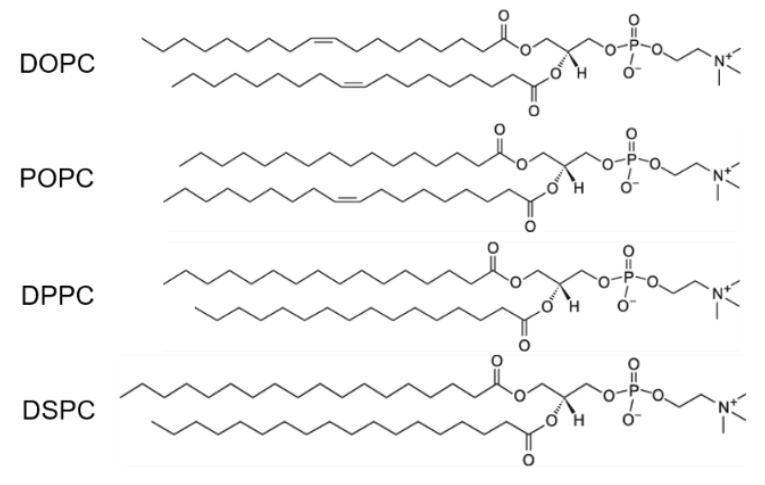

b)

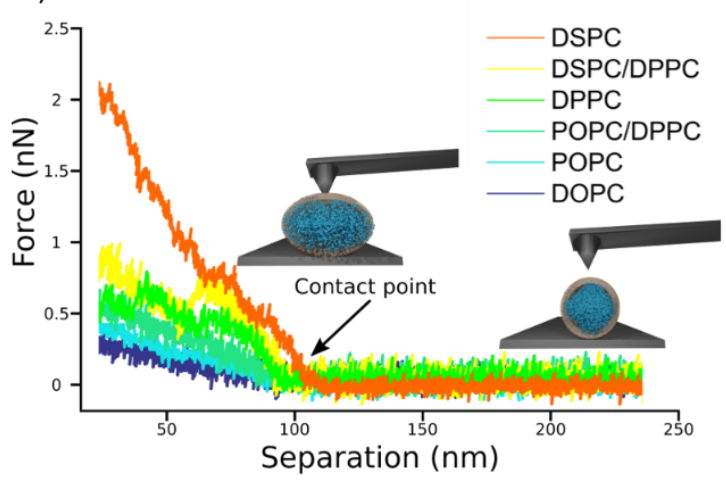

c)

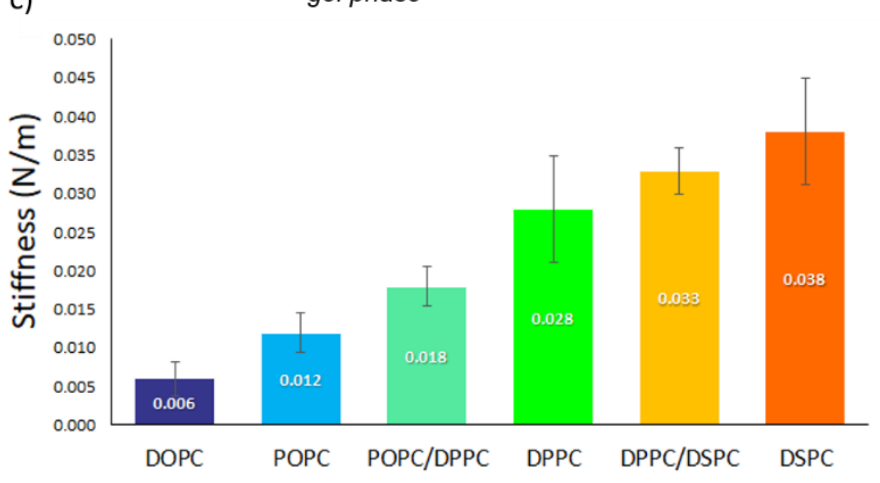

Figure 1. AFM characterization of vesicles stiffnesses. a) Chemical formulas of the four lipids used for the preparation of liposomes (1,2-distearoyl-sn-glycero-3-phosphocholine (DSPC), 1,2-dipalmitoyl-sn-glycero-3-phosphocholine (DPPC), 1-palmitoyl-2-oleoylglycero-3-phosphocholine (POPC) and 1,2-dioleoyl-sn-glycero-3-phosphocholine (DOPC)); depending on the molecular composition, the lipid bilayer enclosing a liposome exhibits a different degree of molecular packing at room temperature, which determines the phase (i.e., fluid or gel) of the membrane. b) AFM force-distances curves for the different vesicles batches, together with graphical representation of vesicles deformation induced by the AFM tip at two different separation distances. Liposomes samples are DOPC; POPC, POPC/DPPC (50/50 mol\%), DPPC, DPPC/DSPC (50/50 mol\%) and DSPC vesicles; c) Stiffness values ( $\mathrm{N} / \mathrm{m}$ ) of the different vesicles, determined through AFM-FS; All error bars represent the uncertainties obtained by bootstrapping (1000 repetitions of 5 draws, with replacement).

Figure $1 \mathrm{~b}$ reports representative AFM-FS force/distance plots of single-vesicle indentation events for each lipid ${ }^{52,53}$. The slope of the linear regime occurring immediately after the contact point represents the stiffness of the vesicles; the stiffnesses in Figure 1c were obtained by averaging the values for multiple vesicles (see Supporting Information for further details). Taken together, the entire series of stiffness values measured on the selected library of synthetic PC standards can be regarded as a stiffness gauge in which the rigidity monotonically increases from DOPC to DSPC vesicles, in line with the literature ${ }^{21,54}$. This set will be used to validate the stiffness plasmon nanoruler. 
The vesicles were then challenged with a water dispersion of negatively charged citrated AuNPs (13 \pm 0.6 $\mathrm{nm}$ diameter, zeta potential: $-36 \pm 2 \mathrm{mV}$ ), and the changes in the SPR profile monitored after 15 minutes (Figure 2a).

The AuNPs dispersion in the absence of lipid vesicles shows a well-defined SPR peak centred at $522 \mathrm{~nm}$ (red curve); upon mixing with liposomes, an immediate colour change is visible to the naked eye (inset, Figure 2a), which clearly depends on the composition of the target membrane. Going from DSPC to DOPC, we observe colour shifts from red to increasingly dark shades of violet and blue. The variation in the SPR profile gradually increases as the stiffness of vesicles decreases. Specifically, from the stiffest vesicles (DSPC) to the softest ones (DOPC), the progressive emergence of a high-wavelength shoulder can be observed, eventually resulting in a secondary plasmon peak at about $625 \mathrm{~nm}$ (see Figure 2a). This new spectral feature is the hallmark of the aggregation of AuNPs, whose spatial proximity produces the coupling of the individual AuNPs plasmons.

a)

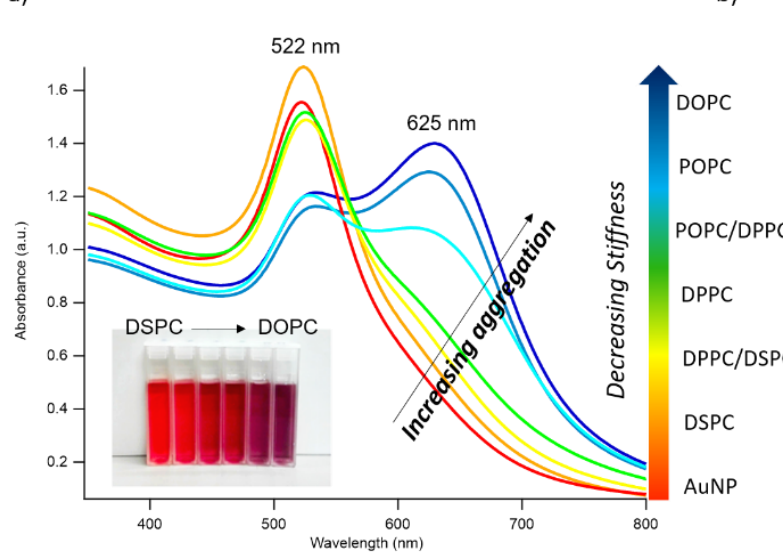

b)

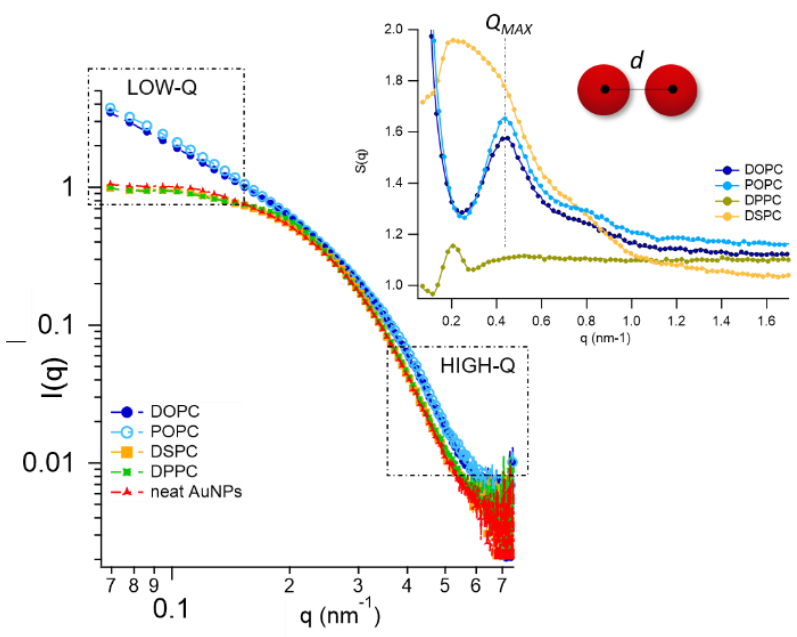

Figure 2. AuNPs interaction with lipid bilayers of different stiffness. a) UV-Vis spectra of AuNPs (1.3 nM) incubated with synthetic vesicles (0.2 nM) (liposomes/AuNPs number ratio 1/100). Inset: visual appearance of the same samples. b) SAXS profiles of NPS with and without vesicles (1:8 vesicles/NPs ratio). Under these conditions, the scattering from vesicles (subtracted from the scattering of AuNPs-vesicles mixtures) is negligible and the observed signal is only due to AuNPs. The power law dependence at low-q is connected to the presence of AuNPs clusters and to their morphology. The power-law exponents for DOPC and POPC, i.e. -1.54 and -1.5 respectively (see Supporting Information), are consistent with an increasing fractal dimension of clusters as the stiffness of vesicles decreases. The absence of such power-law for gel-phase liposomes is related to non-aggregated AuNPs, preserving their original diameter. The right inset is the structure factor $(S(q))$ vs $q$, extracted from the high-q range of vesicles/NPs profiles (see Supporting Information). 
To get insights into the structure of AuNPs aggregates, we performed Small Angle X-ray Scattering (SAXS) for DOPC, POPC, DPPC and DSPC liposomes challenged with AuNPs (Figure 2b).

The power-law dependence in the low-q region highlights the presence of AuNPs clusters on fluid-phase bilayers, with a fractal dimension which increases as the stiffness of vesicles decreases (Figure $2 b$, inset, and Supporting Information) ${ }^{55}$. The spatial correlation between AuNPs was determined from the structure factor $S(q)$, inferred from the high-q region of the scattering profiles (Figure $2 b$, inset, and Supporting Information). The position of the $S(q)$ correlation peaks for fluid-phase liposomes is consistent with NP-NP center-to-center distances comparable with the particle diameter and decreasing with vesicles stiffness (14.5 nm and $14.1 \mathrm{~nm}$ for POPC and DOPC, respectively). For liposomes in the gel phase, no low-q upturn of intensity was detected and the positional correlation corresponds to significantly higher NP-NP distances (i.e., $30.5 \mathrm{~nm}$ and $30.2 \mathrm{~nm}$ for DSPC and DPPC, respectively), hinting at the presence of multiple AuNPs adsorbed on the same liposomal surface, but not aggregated.

According to recent reports, the aggregation of AuNPs on lecithin vesicles is switched on and off by the membrane phase ${ }^{45,46}$ : aggregation is inhibited on gel-phase bilayers (e.g. DPPC and DSPC at r.t.) and promoted by fluid-phase membranes (e.g. DOPC and POPC at r.t.), with no dependence on the molecular composition for bilayers in the same phase ${ }^{45,46}$. Conversely, the UV-Vis and SAXS data here shown provide additional insights, highlighting that -in these experimental conditions- AuNPs clustering on lipid vesicles is not abruptly switched-on by varying the membrane physical state, but is rather modulated by the membrane stiffness in a "continuous fashion".

This dependence can be exploited to set-up a UV-Vis spectroscopic assay to probe the mechanical properties of lipid vesicles. With this aim, we analysed the optical spectra to extract a quantitative descriptor. The so-called "stiffness index", S.I., (see Figure 3a), accounting for the main variations in the AuNPs SPR profile, was used to build-up an empirical 'AuNPs spectral response' vs 'vesicles' stiffness' scale. The S.I. for each AuNPs/vesicles hybrid is calculated dividing the area subtended by the absorbance spectrum in the $560-800 \mathrm{~nm}$ range by the area relative to the total spectral range $(350-800 \mathrm{~nm})$. The results are then normalized for the S.I. of neat AuNPs (which is then equal to 1 by definition) to obtain positive integer values of S.I., which gradually increase with increasing AuNPs aggregation extent. 
(a)

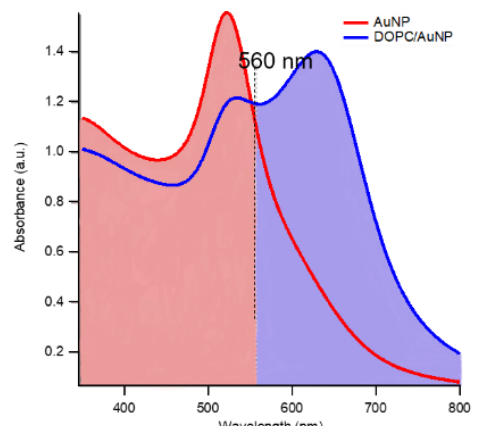

(c)

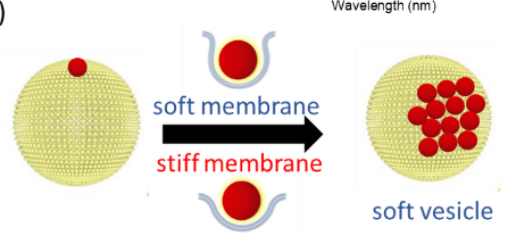

(b)

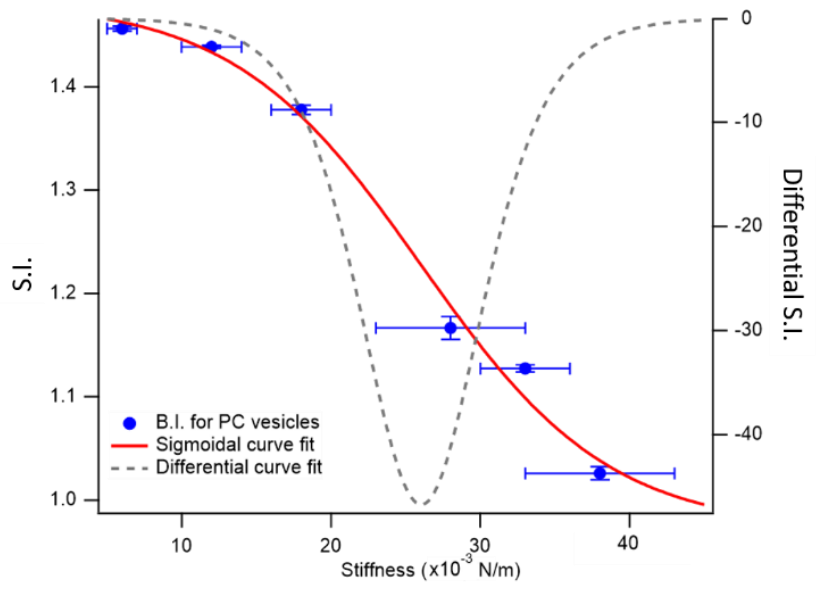

Figure 3. Quantification of Liposomes-induced variation in the AuNPs SPR profile. a) Visual description of the stiffness index (S.I.); b) S.I. values (blue spots) with relative errors bars plotted as a function of membrane stiffness. The red curve is the sigmoidal curve fit, while the grey dashed curve is the first derivative of the sigmoidal curve fit with respect to stiffness (see Supporting Info for details on fitting parameters). c) Mechanism of interaction between AuNPs and vesicles characterized by different stiffness. The adhesion of an AuNP on a soft membrane is followed by a significant AuNP wrapping by the membrane, resulting into AuNPs aggregation on the vesicle surface. The AuNP docking on a stiffer membrane results in a lower wrapping extent, preventing AuNPS clustering.

Figure $3 \mathrm{~b}$ reports the S.I. (blue dots) obtained for the liposome set plotted as a function of vesicles stiffness, obtained from AFM-FS measurements (Figure 1). Each point represents the average value obtained from five independent batches, which highlights a particularly high reproducibility.

The dependence of the S.I. on stiffness can be expressed by a sigmoidal law, with the following expression:

$$
\text { S. I. }=\frac{b}{1+\exp \left(\frac{c-S}{d}\right)}+a
$$

with $\mathrm{S}$ the stiffness obtained from AFM-FS and $a, b, c$ and $d$ constant fitting parameters (see red profile in Figure $3 b$ for the best fitting curve and Supporting Information for further details).

For this set of synthetic vesicles, having superimposable size distributions and a luminal content identical to the external medium, the stiffness differences observed in AFM-FS are only due to a membrane 
contribution, which results from the different composition of the bilayers. As it is well-stablished, the mechanical response of a lipid bilayer is mainly controlled by its bending rigidity ${ }^{21}$, quantified by the bilayer bending modulus. Therefore, in these experimental conditions, it is the bilayer bending modulus that determines the overall stiffness of the vesicles and in turn the extent of AuNPs aggregation (i.e. the S.I.).

Interestingly, in a recent simulation Lipowsky and co-authors ${ }^{56}$ report a sigmoidal correlation between the wrapping efficiency of spherical NPs interacting with model membranes and the bilayer bending modulus. This relation holds for fixed NPs radius and membrane-NPs adhesion energy, which perfectly matches our experimental conditions (i.e. NPs of defined size and vesicles with fixed PC headgroups).

This finding is fully in line with a recent report ${ }^{44}$, where AuNPs wrapping, modulated by the membrane bending modulus, is recognized as the main driver for the membrane-templated aggregation of AuNPs, through the mechanism sketched in Figure 3c.

The dependence of the S.I. on the stiffness of vesicles (eqn. 1) allows a quantitative estimate of the mechanical properties of membrane-enclosed compartments of unknown composition. The method here proposed possess high sensitivity and reproducibility. In fact, it is able to robustly discriminate systems with very close stiffnesses, as POPC and DOPC vesicles, whose mechanical properties are usually not distinguishable with many other techniques ${ }^{54,57}$.

In addition, the presence of a sigmoidal law, which exhibits the highest variation of S.I. in the central region of the selected set of stiffnesses (see grey dashed curve of Figure $3 \mathrm{~b}$, representing the first derivative of the sigmoidal fit) provides maximum sensitivity in the region where the rigidities of natural membranes usually fall (i.e. $0.02-0.025 \mathrm{~N} / \mathrm{m}^{58}$ ).

We chose EVs to further validate the method and to provide evidence of its applicability on membranous nanoparticles which are more challenging both in terms of compositional and structural complexity, as well as in analyte availability. Specifically, we assayed a sample EVs from the murine cell line TRAMP-C2, with size and z-potential similar to the PC synthetic liposomes (see Supporting Information for details). The EVs were separated from the cell culture medium and characterized at the best of the current state of the art ${ }^{59}$, by the protocols described in Paolini et al. (medium EVs) ${ }^{60}$. The morphology of EVs was investigated by liquid imaging AFM (see Figure 4a), showing the characteristic spherical cap shape of EVs adhered onto a substrate and an average diameter of $74.3 \mathrm{~nm}$ (refer to Supporting Info for further details). 

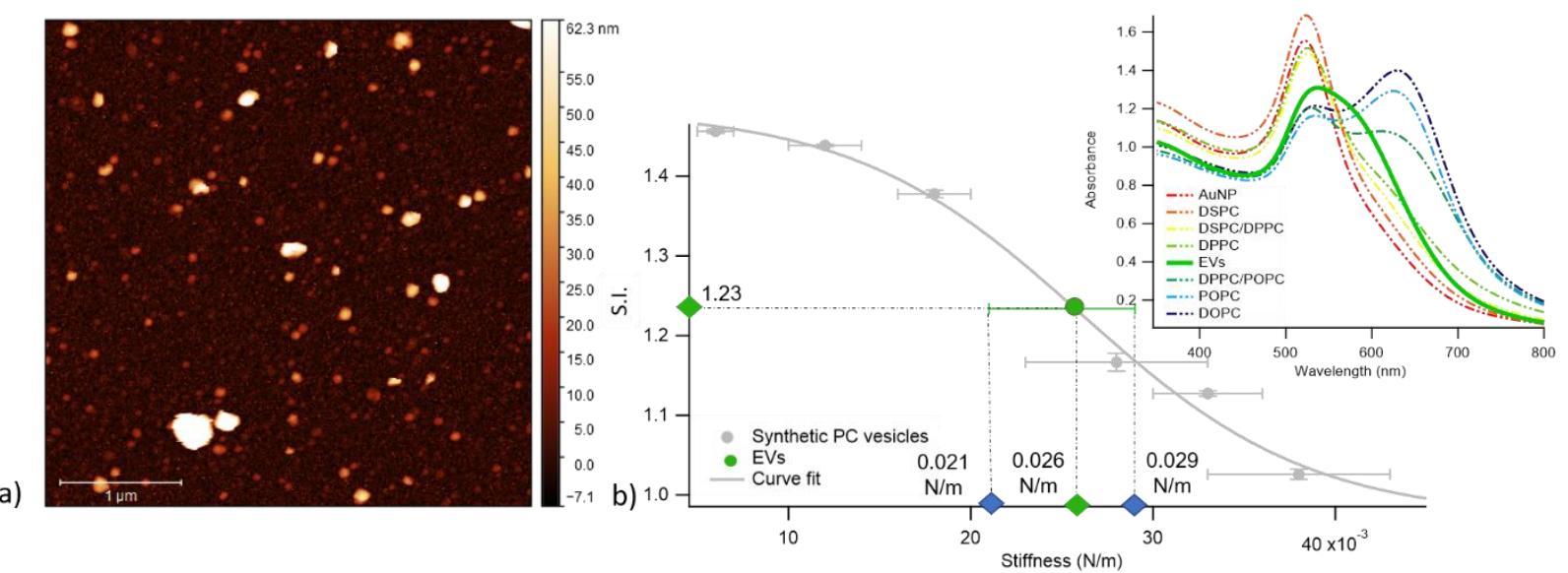

Figure 4. Quantification of Liposomes-induced variation in the SPR profile of AuNPs. a) Representative AFM image of EVs; b) Sigmoidal trend of the S.I. as a function of membrane stiffness. The EVs' S.I. (1.23), evaluated through UV-Vis spectroscopy, and stiffness, predicted by the sigmoidal law $(0.026 \mathrm{~N} / \mathrm{m})$, are reported as green points in the graph. The green error bar represents the stiffness interval obtained through AFM-FS for EVs. The right inset reports the UV-Vis spectra of AuNPs (1.3 nM) in the presence of synthetic PC vesicles (dashed curves) and natural EVs (solid green curve) at a lipid concentration of $0.04 \mathrm{mg} / \mathrm{ml}$.

The stiffness of EVs, determined through AFM-FS as previously described for PC liposomes, falls in the middle of the stiffness interval defined by the synthetic standards used for calibration $(0.025 \pm 0.004 \mathrm{~N} / \mathrm{m})$, in between the values obtained for DPPC and DPPC/POPC vesicles (see Figure 1c). EVs were mixed with AuNPs in the same conditions (AuNPs/vesicles number ratio, incubation time and temperature) previously employed for synthetic liposomes and the SPR profile change of AuNPs was recorded through UV-Vis spectroscopy (right inset of Figure 4b).

In full agreement with the AFM analysis, this SPR profile variation, S.I. $=1.23 \pm 0.01$, is intermediate between the ones of DPPC, S.I. $=1.16 \pm 0.01$, and DPPC/POPC, S.I. $=1.377 \pm 0.005$. This result demonstrates that the correlation between AuNPs aggregation and vesicles stiffness, observed in liposomes, also holds for the case of far more complex nanosized vesicles of biological origin. More importantly, the value of stiffness estimated from the S.I. of the AuNPs/EVs hybrid according to the calibration trend (i.e., $0.0259 \pm$ $0.0005 \mathrm{~N} / \mathrm{m}$ ) falls right in the middle of the EVs stiffness range determined through AFM (Figure $4 \mathrm{~b}$ ): this striking agreement proves the predictive ability of this new optical method, showing that the nanoplasmonic properties of AuNPs can be effectively harnessed to assess the stiffness of membraneconfined objects with high sensitivity. 


\section{Conclusions}

The determination of the stiffness of synthetic and natural vesicles is particularly challenging. Here, we show that the SPR of AuNPs can be exploited to quantify this property: combining UV-Vis spectroscopy, Small Angle X-ray Scattering and AFM-based force spectroscopy, we prove that AuNPs aggregation, induced by the interaction with lipid membranes and quantified by an empirical index S.I., exhibits a clear dependence on the mechanical properties of synthetic vesicles. This dependence, expressed by a sigmoidal law, can be used to estimate the stiffness of biological membrane compartments, e.g. EVs, of unknown composition and properties. Similarly to the plasmon ruler developed by El-Sayed et al. ${ }^{33}$, where the SPR of AuNPs are used to probe their mutual distance, we define a "stiffness nanoruler", where the plasmon resonance is applied to probe the nanomechanics of a target membrane. This method, proposed here for the first time, requires commonly available instrumentation, is very reproducible and sensitive, and permits the analysis on sample quantities as small as $15 \mu \mathrm{l}$ (with a concentration of EVs in the $10^{-8} \mathrm{M}$ range), which represents a game-changer for precious biological samples, otherwise intractable. Moreover, differently from other methods (e.g., AFM, micropipette) which probe the stiffness of single objects, it allows for the determination of ensemble-averaged stiffness, where possible variability across the population is considered.

\section{Conflicts of interest}

There are no conflicts to declare

\section{Acknowledgements}

This work has been supported by the European Community through the evFOUNDRY project (H2020FETopen, ID: 801367). We also acknowledge MIUR-Italy (“Progetto Dipartimenti di Eccellenza 2018-2022, ref B96C1700020008" allocated to Department of Chemistry "Ugo Schiff") for the economic support. We thank the SPM@ISMN research facility for support in the AFM experiments. The European Synchrotron Radiation Facility (ESRF) is acknowledged for provision of beam-time. 
(1) Mendozza, M.; Caselli, L.; Berti, D.; Salvatore, A. Nanoparticles and Organized Lipid Assemblies : From Interaction to Design of Hybrid Soft Devices Inorganic Stimuli Responsive. Soft Matter 2019, 15 (44), 8951-8970. https://doi.org/10.1039/c9sm01601e.

(2) Nel, A. E.; Mädler, L.; Velegol, D.; Xia, T.; Hoek, E. M. V; Somasundaran, P.; Klaessig, F.; Castranova, V.; Thompson, M. Understanding Biophysicochemical Interactions at the Nano-Bio Interface. Nat. Mater. 2009, 8 (7), 543-557. https://doi.org/10.1038/nmat2442.

(3) Beddoes, C. M.; Case, C. P.; Briscoe, W. H. Understanding Nanoparticle Cellular Entry: A Physicochemical Perspective. Adv. Colloid Interface Sci. 2015, 218, 48-68.

https://doi.org/10.1016/j.cis.2015.01.007.

(4) K. Nagayama, P. K. Particles at Fluid Interfaces and Membranes: Attachment of Colloid Particles, 1st Editio.; Elsevier Science, 2001.

(5) Chen, K. L.; Bothun, G. D. Nanoparticles Meet Cell Membranes: Probing Nonspecific Interactions Using Model Membranes. Environ. Sci. Technol. 2014, 48 (2), 873-880. https://doi.org/10.1021/es403864v.

(6) Guo, P.; Liu, D.; Subramanyam, K.; Wang, B.; Auguste, D. T.; Moses, M. A. Nanoparticle Elasticity Directs Tumor Uptake. Nat. Commun. 2018, 9 (130). https://doi.org/10.1038/s41467-017-025889.

(7) Simunovic, M.; Prévost, C.; Bassereau, P.; Bassereau, P. Physical Basis of Some Membrane Shaping Mechanisms Subject Areas : Author for Correspondence : Phil. Trans. R. Soc. A 2016, 374, 20160034.

(8) Canham, P. B. The Minimum Energy of Bending as a Possible Explanation of the Biconcave Shape of the Human Red Blood Cell. J. Theor. Biol. 1970, 26 (1), 61-81. https://doi.org/10.1016/S00225193(70)80032-7.

(9) Evans, E. A. Bending Resistance and Chemically Induced Moments. Biophys. J. 1974, 14, 923-931.

(10) Dumitru, A. C.; Poncin, M.; Conrard, L.; Dufrêne, Y. F.; Tyteca, D.; Alsteens, D. Nanoscale Membrane Architecture of Healthy and Pathological Red Blood Cells. Nanoscale Horizons 2018, 3, 293-304. https://doi.org/10.1039/C7NH00187H.

(11) Safeukui, I.; Buffet, P. A.; Deplaine, G.; Perrot, S.; Brousse, V.; Sauvanet, A.; Aussilhou, B.; David, P. H.; Dokmak, S.; Couvelard, A.; Cazals-hatem, D.; Mercereau-puijalon, O.; Mohandas, N. Sensing of Red Blood Cells with Decreased Membrane Deformability by the Human Spleen. Blood Adv. 2018, 2 (20), 1-4. https://doi.org/10.1182/bloodadvances.2018024562.

(12) Alibert, C.; Goud, B.; Manneville, J. Are Cancer Cells Really Softer than Normal Cells ? Biol. Cell 2017, 109, 167-189. https://doi.org/10.1111/boc.201600078.

(13) Vorselen, D.; van Dommelen, S. M.; Sorkin, R.; Piontek, M. C.; Schiller, J.; Döpp, S. T.; Kooijmans, S. A. A.; van Oirschot, B. A.; Versluijs, B. A.; Bierings, M. B.; van Wijk, R.; Schiffelers, R. M.; Wuite, G. J. L.; Roos, W. H. The Fluid Membrane Determines Mechanics of Erythrocyte Extracellular Vesicles and Is Softened in Hereditary Spherocytosis. Nat. Commun. 2018, 9 (1), 1-9.

(14) Raposo, G.; Stahl, P. D. Extracellular Vesicles: A New Communication Paradigm? Nat. Rev. Mol. Cell Biol. 2019, 20, 509-510. https://doi.org/10.1038/s41580-019-0158-7. 
(15) Niel, G. Van; Angelo, G. D.; Raposo, G. Shedding Light on the Cell Biology of Extracellular Vesicles. Nat Rev Mol Cell Biol. 2018, 19 (4), 213-228. https://doi.org/10.1038/nrm.2017.125.

(16) Yáñez-mó, M.; Al., E. Biological Properties of Extracellular Vesicles and Their Physiological Functions. J Extracell Vesicles 2015, 14 (4), 27066. https://doi.org/10.3402/jev.v4.27066.

(17) Whitehead, B.; Wu, L. P.; Hvam, M. L.; Aslan, H.; Dong, M.; DyrskjØt, L.; Ostenfeld, M. S.; Moghimi, S. M.; Howard, K. A. Tumour Exosomes Display Differential Mechanical and Complement Activation Properties Dependent on Malignant State: Implications in Endothelial Leakiness. J. Extracell. Vesicles 2015, 4 (1), 1-11. https://doi.org/10.3402/jev.v4.29685.

(18) Paolini, L.; Zendrini, A.; Radeghieri, A. Biophysical Properties of Extracellular Vesicles in Diagnostics. Biomark. Med. 2018, 12 (4), 383-391. https://doi.org/10.2217/bmm-2017-0458.

(19) Carrasco, C.; Castellanos, M.; De Pablo, P. J.; Mateu, M. G. Manipulation of the Mechanical Properties of a Virus by Protein Engineering. Proc. Natl. Acad. Sci. U. S. A. 2008, 105 (11), 41504155. https://doi.org/10.1073/pnas.0708017105.

(20) Roos, W. H. AFM Nanoindentation of Protein Shells, Expanding the Approach beyond Viruses. Semin. Cell Dev. Biol. 2018, 73, 145-152. https://doi.org/10.1016/j.semcdb.2017.07.044.

(21) Nagle, J. F.; Jablin, M. S.; Tristram-nagle, S.; Akabori, K. What Are the True Values of the Bending Modulus of Simple Lipid Bilayers ? Chem. Phys. Lipids 2015, 185, 3-10. https://doi.org/10.1016/j.chemphyslip.2014.04.003.

(22) Bochicchio, D.; Monticelli, L. The Membrane Bending Modulus in Experiments and Simulations : A Puzzling Picture, 1st ed.; Elsevier Inc., 2016; Vol. 23. https://doi.org/10.1016/bs.abl.2016.01.003.

(23) Bezlyepkina, N.; Knorr, R. L.; Lipowsky, R.; Dimova, R. Effect of Cholesterol on the Rigidity of Saturated and Unsaturated Membranes : Fluctuation and Electrodeformation Analysis of Giant Vesicles + . Soft Matter 2010, 6, 1472-1482. https://doi.org/10.1039/b920629a.

(24) Henriksen, J. R.; Ipsen, J. H. Measurement of Membrane Elasticity by Micro-Pipette Aspiration. Eur. Phys. J. E 2004, 167, 149-167. https://doi.org/10.1140/epje/i2003-10146-y.

(25) Length, C.; Fragneto, G.; Charitat, T.; Bellet-amalric, E.; Cubitt, R. Swelling of Phospholipid Floating Bilayers : The Effect of Chain Lenght. Langmuir 2003, 19, 7695-7702. https://doi.org/10.1021/la026972x.

(26) Pan, J.; Tristram-nagle, S.; Nagle, J. F. Effect of Cholesterol on Structural and Mechanical Properties of Membranes Depends on Lipid Chain Saturation. Phys. Rev. E 2009, 80, 021931. https://doi.org/10.1103/PhysRevE.80.021931.

(27) Mell, M.; Moleiro, L. H.; Hertle, Y.; Fouquet, P.; Schweins, R.; Hellweg, T.; Monroy, F. Bending Stiffness of Biological Membranes : What Can Be Measured by Neutron Spin Echo ? Eur. Phys. J. E 2013, 36, 75. https://doi.org/10.1140/epje/i2013-13075-2.

(28) Marsh, D. Elastic Curvature Constants of Lipid Monolayers and Bilayers. Chem. Phys. Lipids 2006, 144 (2), 146-159. https://doi.org/10.1016/j.chemphyslip.2006.08.004.

(29) Nagle, J. F. Introductory Lecture : Basic Quantities in Model Biomembranes. Faraday Discuss. 2013, 161, 11-29. https://doi.org/10.1039/c2fd20121f.

(30) Dimova, R. Recent Developments in the Field of Bending Rigidity Measurements on Membranes. 
Advances in Colloid and Interface Science. 2014, pp 225-234. https://doi.org/10.1016/j.cis.2014.03.003.

(31) Piontek, M. C.; Lira, R. B.; Roos, W. H. Active Probing of the Mechanical Properties of Biological and Synthetic Vesicles. Biochim. Biophys. Acta - Gen. Subj. 2019, No. September, 129486. https://doi.org/10.1016/j.bbagen.2019.129486.

(32) Amendola, V.; Pilot, R.; Frasconi, M. Surface Plasmon Resonance in Gold Nanoparticles : A Review. J. Phys. Condens. Matter 2017, 29, 203002. https://doi.org/10.1088/1361-648X/aa60f3.

(33) Jain, P. K.; Huang, W.; El-sayed, M. A. On the Universal Scaling Behavior of the Distance Decay of Plasmon Coupling in Metal Nanoparticle Pairs : A Plasmon Ruler Equation. Nano Lett. 2007, 7 (7), 2080-2088.

(34) Zhao, W.; Ali, M. M.; Aguirre, S. D.; Brook, M. A.; Li, Y. Paper-Based Bioassays Using Gold Nanoparticle Colorimetric Probes. Anal. Chem. 2008, 80 (22), 8431-8437. https://doi.org/10.1021/ac801008q.

(35) Chang, C. C.; Chen, C. P.; Wu, T. H.; Yang, C. H.; Lin, C. W.; Chen, C. Y. Gold Nanoparticle-Based Colorimetric Strategies for Chemical and Biological Sensing Applications. Nanomaterials 2019, 9 (6), 1-24. https://doi.org/10.3390/nano9060861.

(36) Maiolo, D.; Paolini, L.; Di Noto, G.; Zendrini, A.; Berti, D.; Bergese, P.; Ricotta, D. Colorimetric Nanoplasmonic Assay to Determine Purity and Titrate Extracellular Vesicles. Anal. Chem. 2015, 87 (8), 4168-4176. https://doi.org/10.1021/ac504861d.

(37) Zendrini, A.; Paolini, L.; Busatto, S.; Radeghieri, A.; Romano, M.; Wauben, M. H. M.; van Herwijnen, M. J. C.; Nejsum, P.; Borup, A.; Ridolfi, A.; Montis, C.; Bergese, P. Augmented COlorimetric NANoplasmonic (CONAN) Method for Grading Purity and Determine Concentration of EV Microliter Volume Solutions. Front. Bioeng. Biotechnol. 2020, 7 (February), 1-10. https://doi.org/10.3389/fbioe.2019.00452.

(38) Mallardi, A.; Nuzziello, N.; Liguori, M.; Avolio, C.; Palazzo, G. Counting of Peripheral Extracellular Vesicles in Multiple Sclerosis Patients by an Improved Nanoplasmonic Assay and Dynamic Light Scattering. Colloids Surfaces B Biointerfaces 2018, 168, 134-142. https://doi.org/10.1016/j.colsurfb.2018.02.006.

(39) Ridolfi, A.; Caselli, L.; Montis, C.; Mangiapia, G.; Berti, D.; Brucale, M.; Valle, F. Gold Nanoparticles Interacting with Synthetic Lipid Rafts: An AFM Investigation. J. Microsc. 2020, 00 (0), 1-10. https://doi.org/10.1111/jmi.12910.

(40) Montis, C.; Maiolo, D.; Alessandri, I.; Bergese, P.; Berti, D. Interaction of Nanoparticles with Lipid Membranes: A Multiscale Perspective. Nanoscale 2014, 6 (12), 6452-6457. https://doi.org/10.1039/c4nr00838c.

(41) Montis, C.; Generini, V.; Boccalini, G.; Bergese, P.; Bani, D.; Berti, D. Model Lipid Bilayers Mimic Non-Specific Interactions of Gold Nanoparticles with Macrophage Plasma Membranes. J. Colloid Interface Sci. 2018, 516, 284-294. https://doi.org/10.1016/j.jcis.2018.01.064.

(42) Liu, J. Interfacing Zwitterionic Liposomes with Inorganic Nanomaterials: Surface Forces, Membrane Integrity, and Applications. Langmuir 2016, 32 (18), 4393-4404. https://doi.org/10.1021/acs.langmuir.6b00493. 
(43) Wang, F.; Liu, J. Self-Healable and Reversible Liposome Leakage by Citrate-Capped Gold Nanoparticles: Probing the Initial Adsorption/Desorption Induced Lipid Phase Transition. Nanoscale 2015, 7 (38), 15599-15604. https://doi.org/10.1039/C5NR04805B.

(44) Montis, C.; Caselli, L.; Valle, F.; Zendrini, A.; Carlà, F.; Schweins, R.; Maccarini, M.; Bergese, P.; Berti, D. Shedding Light on Membrane-Templated Clustering of Gold Nanoparticles. J. Colloid Interface Sci. 2020, 573, 204-214. https://doi.org/10.1016/j.jcis.2020.03.123.

(45) Sugikawa, K.; Kadota, T.; Yasuhara, K.; Ikeda, A. Anisotropic Self-Assembly of Citrate-Coated Gold Nanoparticles on Fluidic Liposomes. Angew. Chemie - Int. Ed. 2016, 55 (12), 4059-4063. https://doi.org/10.1002/anie.201511785.

(46) Wang, F.; Curry, D. E.; Liu, J. Driving Adsorbed Gold Nanoparticle Assembly by Merging Lipid Gel/Fluid Interfaces. Langmuir 2015, 31 (49), 13271-13274.

https://doi.org/10.1021/acs.langmuir.5b03606.

(47) Sorkin, R.; Huisjes, R.; Boškovic, F.; Vorselen, D.; Pignatelli, S.; Ofir-birin, Y.; Leal, J. K. F.; Schiller, J.; Mullick, D.; Roos, W. H.; Bosman, G.; Regev-rudzki, N.; Schiffelers, R. M.; Wuite, G. J. L. Nanomechanics of Extracellular Vesicles Reveals Vesiculation Pathways. Small 2018, 1801650, 18. https://doi.org/10.1002/smll.201801650.

(48) Nagle, J. F.; Pan, J.; Tristram-nagle, S.; Kuc, N. Temperature Dependence of Structure, Bending Rigidity, and Bilayer Interactions of Dioleoylphosphatidylcholine Bilayers. Biophys J. 2008, 94, 117-124. https://doi.org/10.1529/biophysj.107.115691.

(49) Dimova, R.; Pouligny, B.; Dietrich, C. Pretransitional Effects in Dimyristoylphosphatidylcholine Vesicle Membranes : Optical Dynamometry Study. Biophys. J. 2000, 79 (1), 340-356. https://doi.org/10.1016/S0006-3495(00)76296-5.

(50) Lee, C.; Lin, W.; Wang, J. All-Optical Measurements of the Bending Rigidity of Lipid-Vesicle Membranes across Structural Phase Transitions. Phys. Rev. E 2001, 64, 020901. https://doi.org/10.1103/PhysRevE.64.020901.

(51) Mecke, K. R.; Charitat, T. Fluctuating Lipid Bilayer in an Arbitrary Potential: Theory and Experimental Determination of Bending Rigidity. Langmuir 2003, 19, 2080-2087. https://doi.org/10.1021/la026606d.

(52) Afm, U. C.; Sharma, S.; Rasool, H. I.; Palanisamy, V.; Mathisen, C.; Schmidt, Ḱ. M.; Wong, D. T.; Gimzewski, J. K. Structural-Mechanical Characterization. ACS Nano 2010, 4 (4), 1921-1926. https://doi.org/10.1021/nn901824n.

(53) Krieg, M.; Fl, G.; Alsteens, D.; Gaub, B. M.; Roos, W. H.; Wuite, G. J. L.; Gaub, H. E.; Gerber, C.; Dufr, Y. F. Atomic Force Microscopy- Based Mechanobiology. Nat. Rev. Phys. 2019, 1, 41-57. https://doi.org/10.1038/s42254-018-0001-7.

(54) Nagle, J. F. Experimentally Determined Tilt and Bending Moduli of Single-Component Lipid Bilayers. Chem. Phys. Lipids 2017, 205, 18-24. https://doi.org/10.1016/j.chemphyslip.2017.04.006.

(55) Feigin, L. A.; Svergun, D. S. Structure Anaysis by Small Angle X-Ray and Neutron Scattering; Taylor, G. W., Ed.; Plenum Press, New York: Princeton, 1987.

(56) Raatz, M.; Lipowsky, R.; Weikl, T. R. Cooperative Wrapping of Nanoparticles by Membrane Tubes. 
Soft Matter 2014, 10 (20), 3570-3577. https://doi.org/10.1039/c3sm52498a.

(57) Niggemann, G.; Kummrow, M.; Helfrich, W.; Niggemann, G.; Kummrow, M.; The, W. H.; Rigidity, B. The Bending Rigidity of Phosphatidylcholine Bilayers : Dependences on Experimental Method, Sample Cell Sealing and Temperature To Cite This Version : HAL Id : Jpa-00248169. J. Phys. II Fr. 1995, 5, 413-425.

(58) Ridolfi, A.; Brucale, M.; Montis, C.; Caselli, L.; Paolini, L.; Borup, A.; Boysen, A. T.; Loria, F.; Van Herwijnen, M. J. C.; Kleinjan, M.; Nejsum, P.; Zarovni, N.; Wauben, M. H. M.; Berti, D.; Bergese, P.; Valle, F. AFM-Based High-Throughput Nanomechanical Screening of Single Extracellular Vesicles. Anal. Chem. 2020, 92 (15), 10274-10282. https://doi.org/10.1021/acs.analchem.9b05716.

(59) Théry, C.; Al., E. Minimal Information for Studies of Extracellular Vesicles 2018 (MISEV2018): A Position Statement of the International Society for Extracellular Vesicles and Update of the MISEV2014 Guidelines. J. Extracell. Vesicles 2018, 7 (1), 1535750.

https://doi.org/10.1080/20013078.2018.1535750.

(60) Paolini, L.; Federici, S.; Consoli, G.; Arceri, D.; Radeghieri, A.; Alessandri, I.; Bergese, P. FourierTransform Infrared (FT-IR) Spectroscopy Fingerprints Subpopulations of Extracellular Vesicles of Different Sizes and Cellular Origin. J. Extracell. Vesicles 2020, 9 (1), 1741174.

\section{Supporting Information}

\section{Supplementary Materials and Methods}

\section{Materials}

Tetrachloroauric (III) acid ( $\geq 99.9 \%$ ) and trisodium citrate dihydrate ( $\geq 99.9 \%)$ for the synthesis of AuNPs were provided by Sigma-Aldrich (St. Louis, MO). 1,2-dioleoyl-sn-glycero-3-phosphocholine (DOPC) (>99\%), 1-palmitoyl-2-oleoyl-sn-glycero-3-phosphocholine (POPC) $(\geq 98.0 \%)$, 1,2-dipalmitoyl-sn-glycero-3phosphocholine (DPPC) (>99\%) and 1,2-distearoyl-sn-glycero-3-phosphocholine (DSPC) (>99\%) for the liposomes preparation were provided by Sigma-Aldrich (St. Louis, MO). All chemicals were used as received. Milli-Q grade water was used in all preparations.

\section{Synthesis of citrated AuNPs}

Anionic gold nanospheres of $16 \mathrm{~nm}$ in size were synthesized according to the Turkevich-Frens method ${ }^{1,2}$. Briefly, $20 \mathrm{~mL}$ of a $1 \mathrm{mM} \mathrm{HAuCl} 4$ aqueous solution was brought to boiling temperature under constant and vigorous magnetic stirring. $2 \mathrm{~mL}$ of $1 \%$ citric acid solution were then added and the solution was further boiled for 20 minutes, until it acquired a deep red color. The nanoparticles dispersion was then slowly cooled down to room temperature.

\section{Preparation of liposomes}


The proper amount of lipid was dissolved in chloroform and a lipid film was obtained by evaporating the solvent under a stream of nitrogen and overnight vacuum drying. The film was then swollen and suspended in warm $\left(50^{\circ} \mathrm{C}\right)$ milliQ-water by vigorous vortex mixing, in order to obtain a final $4 \mathrm{mg} / \mathrm{ml} \mathrm{lipid}$ concentration. The resultant multilamellar liposomes in water were subjected to 10 freeze-thaw cycles and extruded 10 times through two stacked polycarbonate membranes with $100 \mathrm{~nm}$ pore size at room temperature, to obtain unilamellar liposomes with narrow and reproducible size distribution. The filtration was performed with the Extruder (Lipex Biomembranes, Vancouver (Canada)) through Nuclepore membranes.

\section{Preparation of liposomes/AuNPs hybrids}

The hybrid samples preparation procedure for Figure 2a of the main text is the following: $20 \mu \mathrm{L}$ of liposomes (previously diluted to a final lipid concentration of $0.04 \mathrm{mg} / \mathrm{ml}$ ) or extracellular vesicles were placed inside a $500 \mu \mathrm{L}$ UV-Vis plastic cuvette. Then $100 \mu \mathrm{L}$ of citrated gold nanoparticles $\left(6.7 \cdot 10^{-9} \mathrm{M}\right.$, see "Synthesis citrated Gold Nanoparticles" and "Supplementary Characterization of Gold Nanoparticles" of $\mathrm{SI}$ ) were added, in order to have a final concentration (inside the cuvette) of $\sim 5 \cdot 10^{-11} \mathrm{M}$ and of $\sim 5 \cdot 10^{-9} \mathrm{M}$ for liposomes and AuNPs, respectively, and AuNPs/liposomes number ratio of $\sim 100$. Samples were incubated for 15 minutes, then the UV-Vis spectra were recorded.

The hybrid samples preparation procedure for Figure $2 b$ of the main text is the following: fixed volumes $\left(768.9 \mu \mathrm{L}\right.$ ) of AuNPs dispersion $\left(6.7 \cdot 10^{-9} \mathrm{M}\right)$ were added to $20 \mu \mathrm{L}$ of liposomes (see Table S4 of SI for liposomes concentration), in order to have a final AuNPs/liposomes number ratio of $\sim 8$. Samples were incubated for 15 minutes, then placed in glass capillaries of $1 \mathrm{~mm}$ diameter and Small-Angle X-Ray profiles acquired.

\section{UV-vis spectroscopy}

UV-Vis spectra were measured with a JASCO UV-Vis spectrophotometer.

\section{Small Angle X-ray Scattering}

SAXS measurements for the characterization of AuNPs were carried out on a S3-MICRO SAXS/WAXS instrument (HECUS GmbH, Graz, Austria) which consists of a GeniX microfocus X-ray sealed Cu Ka source (Xenocs, Grenoble, France) of $50 \mathrm{~W}$ power which provides a detector focused X-ray beam with $\lambda=0.1542$ $\mathrm{nm} \mathrm{Cu} \mathrm{Ka} \mathrm{line.} \mathrm{The} \mathrm{instrument} \mathrm{is} \mathrm{equipped} \mathrm{with} \mathrm{two} \mathrm{one-dimensional} \mathrm{(1D)} \mathrm{position} \mathrm{sensitive} \mathrm{detectors}$ (HECUS 1D-PSD-50 M system). Each detector is $50 \mathrm{~mm}$ long (spatial resolution $54 \mu \mathrm{m} /$ channel, 1024 channels) and covers the SAXS q-range $\left(0.003<q<0.6 \AA^{-1}\right)$. The temperature was controlled by means of a Peltier TCCS-3 Hecus. The analysis of SAXS curves was carried out using Igor Pro. ${ }^{3}$ SAXS measurement on AuNPs aqueous dispersion was carried out in a sealed glass capillary of $1.5 \mathrm{~mm}$ diameter. To analyze gold nanospheres' curves we chose a model function with a spherical form factor and a Schulz size distribution: ${ }^{4}$, it calculates the scattering for a polydisperse population of spheres with uniform scattering length density. The distribution of radii is a Schulz distribution given by the following equation: 


$$
f(R)=(z+1)^{z+1} x^{z} \frac{\exp [-(z+1) x]}{R_{\text {avg }} \Gamma(z+1)}
$$

where $R_{\text {avg }}$ is the mean radius, $x=R / R_{\text {avg }}$ and $z$ is related to the polydispersity. The form factor is normalized by the average particle volume, using the $3 r d$ moment of $R$ :

$$
\langle V\rangle=\frac{4 \pi}{3}\left\langle R^{+3}\right\rangle=\frac{4 \pi}{3}\langle R\rangle^{+3} \frac{(z+3)(z+2)}{(z+1)^{2}}
$$

The scattering intensity is:

$$
I(q)=\left(\frac{4 \pi}{3}\right)^{2} N_{0} \Delta \rho^{2} \int_{0}^{\infty} f(R) R^{6} F^{2}(q R) d R
$$

where $N_{0}$ is the total number of particles per unit volume, $F(R)$ is the scattering amplitude for a sphere and $\Delta \rho$ is the difference in scattering length density between the particle and the solvent.

SAXS measurements for the characterization of AuNPs/liposomes hybrids were collected at beamline ID02 at the European Synchrotron Radiation Facility (ESRF, Grenoble, France) ${ }^{5}$. A scattering vector (of magnitude q) range of $0.007 \leq \mathrm{q} \leq 0.2 \mathrm{~nm}^{-1}$ was covered with two sample-detector distances ( 1 and $10 \mathrm{~m}$ ) and a single-beam setting for an X-ray monochromatic radiation wavelength with a wavelength of $\lambda=0.10$ $\mathrm{nm}(12.46 \mathrm{keV})$. The beam diameter was adjusted to $72.4 \mu \mathrm{m}$ in the horizontal ( $\mathrm{x}$ ) direction and $42.3 \mu \mathrm{m}$ in the vertical ( $y$ ) direction (full width at half-maximum at the sample). Assuming a Gaussian distribution, the portion of the beam that is hitting outside the channel can be estimated. When the channel is centered, this is $\sim 0.3 \%$ but closer to the edge and more beam overlaps the edge. The beamstop diameter was $2 \mathrm{~mm}$. As a detector, a 2D Rayonix MX-170HS with a pixel size of $44 \times 44 \mu \mathrm{m}^{2}$ was used, which was housed in an evacuated flight tube, at a sample-to-detector distance of alternatively $10 \mathrm{~m}$ (leading to an available q-range of $0.007-0.02 \mathrm{~nm}^{-1}$ ) or $1 \mathrm{~m}$ (leading to an available q-range of $0.07-0.2 \mathrm{~nm}^{-1}$ ). The exposure times for the background- and sample measurements were $0.5 \mathrm{~s}$ for the case of $1 \mathrm{~m}$ sample-todetector distance and $0.3 \mathrm{~s}$ for the case of $10 \mathrm{~m}$ sample-to-detector distance. Measured scattering patterns were normalized to an absolute intensity scale after applying standard detector corrections and then azimuthally averaged to obtain the one-dimensional intensity profiles, denoted by I (q).

\section{Atomic Force Microscopy (AFM)}

\section{Surface Preparation and Sample Deposition}

All AFM experiments were performed on poly-L-lysine (PLL) coated glass coverslips. All reagents were acquired from Sigma-Aldrich Inc (www.sigmaaldrich.com) unless otherwise stated. Microscopy borosilicate glass slides (15mm diameter round coverslips, Menzel Gläser) were first immersed in a 3:1 mixture of $96 \% \mathrm{H} 2 \mathrm{SO} 4$ and $50 \%$ aqueous $\mathrm{H} 2 \mathrm{O} 2$ ('oxidising piranha') solution for $2 \mathrm{~h}$ in order to remove any organic residue present on their surface; after that, they were cleaned in a sonicator bath (Elmasonic Elma $\mathrm{S} 3 \mathrm{OH}$ ) for 30 minutes in acetone, followed by 30 minutes in isopropanol and 30 minutes in ultrapure water (Millipore Simplicity UV). Clean slides were incubated overnight in a $0.0001 \%(\mathrm{w} / \mathrm{v})$ PLL solution at room temperature, thoroughly rinsed with ultrapure water and dried with nitrogen. A $10 \mu \mathrm{l}$-droplet of the vesicle-containing solution under study was deposited on a PLL-functionalized glass slide and left to 
adsorb for 10 minutes at $4^{\circ} \mathrm{C}$, then inserted in the AFM fluid cell (see below) without further rinsing. The concentration of each vesicle-containing solution was adjusted in order to maximize the surface density of isolated, individual vesicles and minimize clusters of adjoining vesicles.

\section{AFM Setup}

All AFM experiments were performed in ultrapure water at room temperature on a Bruker Multimode (equipped with Nanoscope $V$ electronics, a sealed fluid cell and a type JV piezoelectric scanner) using Bruker SNL-A probes (triangular cantilever, nominal tip curvature radius 2-12 $\mathrm{nm}$, nominal elastic constant $0.35 \mathrm{~N} / \mathrm{m}$, calibrated with the thermal noise method.

\section{AFM Imaging}

Imaging was performed in PeakForce mode. In order to minimize vesicle deformation or rupture upon interaction with the probe, the applied force setpoint was kept in the $150-250 \mathrm{pN}$ range. Lateral probe velocity was not allowed to exceed $5 \mu \mathrm{m} / \mathrm{s}$. Feedback gain was set at higher values than those usually employed for optimal image quality in order to ensure minimal probe-induced vesicle deformation upon lateral contact along the fast scan axis (please refer to Ridolfi et al. ${ }^{6}$ for further details). The average height value of all bare substrate zones was taken as the baseline zero height reference. Image background subtraction was performed using Gwyddion 2.53.16 7 .

\section{AFM-based Force Spectroscopy (AFM-FS)}

The mechanical characterization of vesicles via AFM force spectroscopy was performed by first scanning the sample (see previous paragraph) to locate individual vesicles. The chosen vesicles were then imaged reducing the scan size for achieving higher accuracy. We recorded a series of force/distance curves at multiple XY positions (typically around 64-100 curves arranged in a square array covering the vesicle initial location) for each individual vesicle. In most cases, only a few curves showed the mechanical fingerprint of an intact vesicle response to indentation: a linear deformation upon applied pressure during probe penetration. Of these, we first discarded those curves with probe-vesicle contact points occurring at probe-surface distances below vesicle height as measured by imaging. Remaining traces (typically 1-3 per vesicle) were analyzed to calculate vesicle stiffness (kS).

\section{Transmission Electron Microscopy}

Transmission electron microscopy (TEM) images were acquired with a STEM CM12 Philips electron microscope equipped with an OLYMPUS Megaview G2 camera, at CeME (CNR Florence Research Area, Via Madonna del Piano, 10 - 50019 Sesto Fiorentino). A drop of citrated AuNPs, diluted ten times, was placed on 200 mesh carbon-coated copper grids with a diameter of $3 \mathrm{~mm}$ and a thickness of $50 \mu \mathrm{m}$ (Agar Scientific) and dried at room temperature. Then, the sample was analyzed at an accelerating voltage of $100 \mathrm{keV}$.

\section{Dynamic Light Scattering}


DLS measurements at $\theta=90^{\circ}$ were performed using a Brookhaven Instrument 90 Plus (Brookhaven, Holtsville, NY). Each measurement was an average of ten repetitions of one minute each and repeated ten times. The autocorrelation functions were analyzed through the cumulant fitting stopped to the second order or with Laplace inversion according to CONTIN algorithm, allowing an estimate of the hydrodynamic diameter of particles.

\section{Z-Potential Measurements}

Zeta potential measurements were performed using a Zeta Potential Analyzer (Zeta Plus, Brookhaven Instruments Corporation, Holtsville, NY). Zeta potentials were obtained from the electrophoretic mobility $u$, according to Helmholtz-Smoluchowski equation: $\zeta=(\eta / \varepsilon) \times u$ with $\eta$ being the viscosity of the medium, $\varepsilon$ the dielectric permittivity of the dispersing medium. The Zeta Potential values are reported as averages from ten measurements.

\section{Supplementary Characterization of Gold Nanoparticles}

\section{Transmission Electron Microscopy}

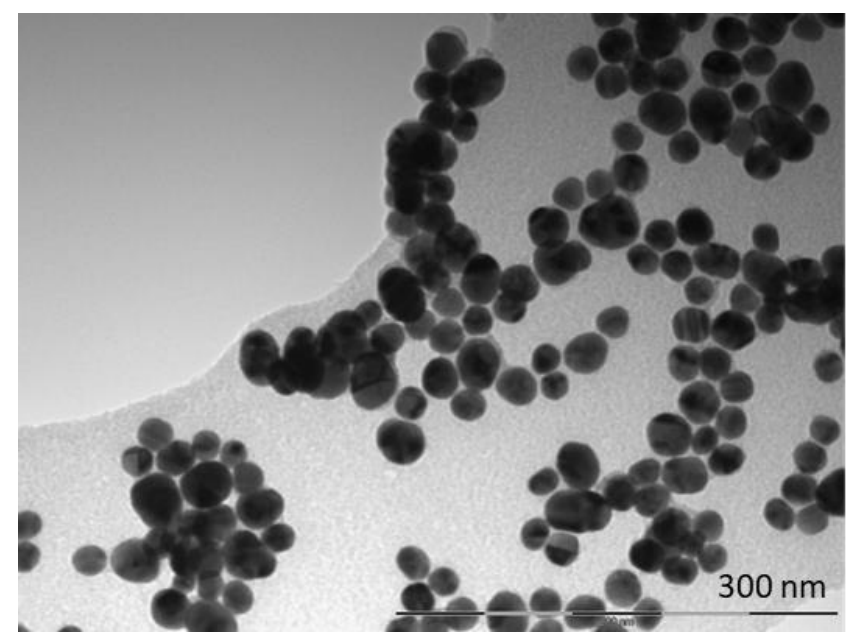

Figure S1 Representative Transmission electron microscopy (TEM) images of citrated gold nanoparticles acquired with a STEM CM12 Philips electron microscope, at CeME (CNR Florence Research Area, Via Madonna del Piano, 10 50019 Sesto Fiorentino). The sample was placed on a 200 mesh carbon-coated copper grid.

\section{Small Angle X-ray Scattering}

The analysis of SAXS curves was carried out using Igor Pro. ${ }^{3}$ SAXS measurements on AuNPs aqueous dispersion were carried out in sealed glass capillaries of $1.5 \mathrm{~mm}$ diameter. To analyze gold nanospheres' curves we chose a model function with a spherical form factor and a Schulz size distribution: ${ }^{4}$, it calculates 
the scattering for a polydisperse population of spheres with uniform scattering length density. The distribution of radii is a Schulz distribution given by the following equation:

$$
f(R)=(z+1)^{z+1} x^{z} \frac{\exp [-(z+1) x]}{R_{\text {avg }} \Gamma(z+1)}
$$

where $R_{\text {avg }}$ is the mean radius, $x=R / R_{\text {avg }}$ and $z$ is related to the polydispersity. The form factor is normalized by the average particle volume, using the $3 \mathrm{rd}$ moment of $R$ :

$$
\langle V\rangle=\frac{4 \pi}{3}\left\langle R^{+3}\right\rangle=\frac{4 \pi}{3}\langle R\rangle^{+3} \frac{(z+3)(z+2)}{(z+1)^{2}}
$$

The scattering intensity is:

$$
I(q)=\left(\frac{4 \pi}{3}\right)^{2} N_{0} \Delta \rho^{2} \int_{0}^{\infty} f(R) R^{6} F^{2}(q R) d R
$$

where $N_{0}$ is the total number of particles per unit volume, $F(R)$ is the scattering amplitude for a sphere and $\Delta \rho$ is the difference in scattering length density between the particle and the solvent. The structural parameters (Table S1) of citrated gold nanoparticles were evaluated from the SAXS profile of their water dispersion (Figure S2) according to the models reported in the Materials and Methods section of SI.

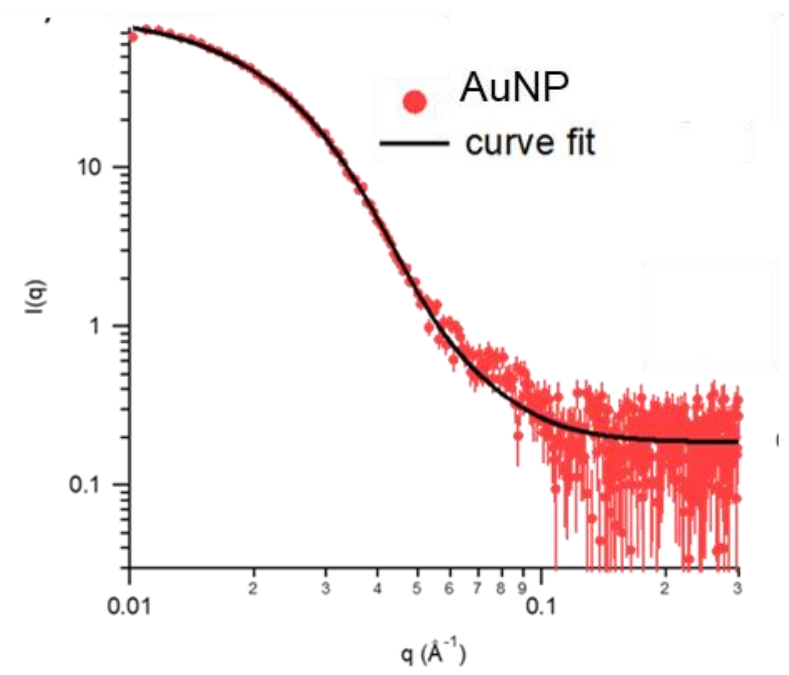

Figure S2 Experimental SAXS curve (red markers) obtained for AuNPs and curve fit (solid black line) according to the Schulz spheres model from the NIST package SANS Utilities. The size and polydispersity obtained from the fitting procedure are summarized in the Table $\mathrm{S} 1$ below. 


\begin{tabular}{lcc}
\hline & Rcore (nm) & poly \\
\hline AuNP & 6.5 & 0.3 \\
\hline
\end{tabular}

Table S1 Structural parameters of the nanoparticles obtained from the analysis of SAXS curves according to the the Schulz spheres model.

\section{Dynamic Light Scattering and Z-Potential}

AuNPs hydrodynamic diameter and surface charge in MilliQ water were evaluated through Dynamic Light Scattering and Zeta-Potential, respectively, and reported in Table S2.

\section{Dh $_{\mathrm{h}}(\mathrm{nm}) \quad$ Z-Potential $(\mathrm{mV})$}

\section{AuNPs $15.8 \pm 0.3 \quad-36 \pm 2$}

Table S2 Hydrodynamic diameter obtained from Dynamic Light Scattering and Zeta Potential values of AuNPs.

\section{UV-vis Spectroscopy}

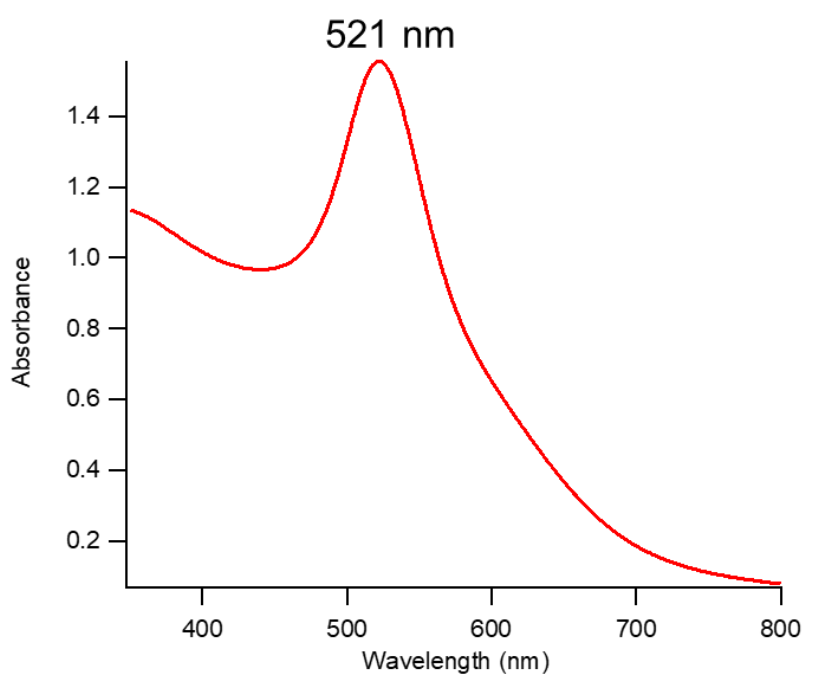

Figure S3 UV-Vis absorption spectra of AuNPs after 1:5 dilution in water. The plasmon absorption peak is at around $521 \mathrm{~nm}$.

The size of AuNPs was further evaluated from UV-Vis Spectroscopy by the following equation ${ }^{8}$ : 


$$
d=\exp \left(B_{1} \frac{A_{s p r}}{A_{450}}-B_{2}\right)
$$

with $d$ diameter of gold nanoparticles, $A_{s p r}$ absorbance at the surface plasma resonance peak, $A_{450}$ absorbance at the wavelength of $450 \mathrm{~nm}$ and $B_{1}$ and $B_{2}$ are dimensionless parameters, taken as 3 and 2.2 , respectively. The diameter value obtained is of $13.5 \mathrm{~nm}$.

The concentration of citrated gold nanoparticles was determined via UV-Vis spectrometry, using the Lambert-Beer law $(E(\lambda)=\varepsilon(\lambda) / c)$, taking the extinction values $E(\lambda)$ at the LSPR maximum, i.e. $\lambda=521 \mathrm{~nm}$. The extinction coefficient $\varepsilon(\lambda)$ of gold nanoparticles dispersion was determined by the method reported in literature ${ }^{9}$, by the following equation:

$$
\ln (\varepsilon)=k \ln (d)+a
$$

with $d$ core diameter of nanoparticles, and $k$ and $a$ dimensionless parameters $(k=3.32111$ and $a=$ 10.80505). The arithmetic mean of the sizes obtained by optical and scattering analyses was selected, leading to a $\varepsilon(\lambda)$ of $2.8 \cdot 10^{8} \mathrm{M}^{-1} \mathrm{~cm}^{-1}$. The final concentration of the citrated AuNPs is therefore $\sim 5.6 \cdot 10^{-9} \mathrm{M}$.

\section{Supplementary Characterization of Liposomes}

\section{Dynamic Light Scattering and Zeta-Potential}

\begin{tabular}{lll}
\hline & $\mathrm{D}_{\mathrm{h}}(\mathrm{nm})$ & Zeta \\
\hline DOPC & $118.6 \pm 0.2$ & $-16 \pm 1$ \\
POPC & $103.8 \pm 0.1$ & $-19 \pm 3$ \\
POPC/DPPC & $92.1 \pm 0.2$ & $-22 \pm 1$ \\
DPPC & $115.7 \pm 0.1$ & $-13 \pm 1$ \\
DPPC/DSPC & $104 \pm 0.2$ & $-10 \pm 1$ \\
DSPC & $127.7 \pm 0.2$ & $-19 \pm 1$ \\
\hline
\end{tabular}

Table S3 Hydrodynamic diameter obtained from Dynamic Light Scattering and Zeta Potential values of synthetic liposomes. 


\section{Evaluation of Liposomes concentration}

The lipid concentration in the starting colloidal dispersion was estimated to be $4 \mathrm{mg} / \mathrm{mL}$ from the initial lipid and water amounts employed in the formation and swelling of lipid films (see "Preparation of liposomes" in the Materials and Methods section), assuming the absence of lipid loss due to the extrusion procedure. The liposomes concentration in the final dispersion was subsequently calculated considering the hydrodynamic diameter of each liposomal batch (Table S3 of SI). In particular, from the liposomes' average diameter, the liposomal surface area (surface area $=4 \pi r^{2}$ ) can be calculated; the doubled surface can be subsequently divided by the lipid cross section $\left(0.5 \mathrm{~nm}^{2}\right)$ in order to obtain the lipid number per liposome, assuming that approximately one half of the lipids is localized in the external leaflet of a liposomes, since the bilayer thickness, about 4-5 nm, is negligible with respect to the liposomes' average diameter. Eventually, the total weighted lipid concentration was divided by the total number of lipids per liposome, yielding the real liposome concentration, which is reported in Table S4 for each liposomes' dispersion.

\begin{tabular}{ll}
\hline & Concentration (M) \\
\hline DOPC & $3.2 \cdot 10^{-8}$ \\
POPC & $3.1 \cdot 10^{-8}$ \\
POPC/DPPC & $3.6 \cdot 10^{-8}$ \\
DPPC & $3.1 \cdot 10^{-8}$ \\
DPPC/DSPC & $3.8 \cdot 10^{-8}$ \\
DSPC & $3.5 \cdot 10^{-8}$ \\
\hline
\end{tabular}

Table S4 Final liposomes' concentration in each liposomal batch.

\section{Supplementary Characterization of EVs}

\section{Zeta Potential}

The Zeta Potential of EVs dispersion in milliQ water was measured as described in the "Material and Methods" section of SI and is equal to $-21 \pm 3 \mathrm{mV}$. 


\section{AFM characterization of synthetic and natural lipid vesicles}

\section{AFM Mechanical Characterization}

According to the Canham-Helfrich theory, the mechanical response of a vesicle to an applied force is elastic; this behavior is reflected in the linear relationship between the force and tip penetration, in the AFM force-distance curves, right after the contact point (see Fig.1b in the main text). Calculating the slope of this linear part, gives the value of the vesicle stiffness, a mechanical parameter that accounts for multiple contributions, the most relevant being the intrinsic membrane rigidity (the bending modulus) and the vesicle luminal, i.e. internal, pressure. The latter contribution describes the vesicle pressurization following the deformation applied by the AFM tip. This deformation generates a volume variation that increases the pressure within the vesicle. While the bending modulus is an intrinsic descriptor of the lipid membrane bending rigidity, the internal pressure and hence the stiffness depend on the size of each vesicle. Indeed, the volume variation associated with a given tip penetration varies with the vesicle size (i.e. the same penetration will result in higher volume variations for smaller vesicles); as a consequence, vesicles that are heterogenous in size will be subjected to different pressurizations following similar indentation events. However, both the measured liposomes and EVs are characterized by low polydispersity, this allows considering the stiffness a size-independent parameter. Moreover, since all the tested liposomes were characterized by similar size distributions and same polar headgroups, they will experience similar pressurizations and electrostatic attractions to the substrate; as a result, we can assume that changes in their stiffness are entirely ascribable to differences in their membrane rigidity, which can be recapitulated by the bending modulus. Membrane rigidity may vary depending on the phase behavior of the lipid bilayer, a temperature dependent parameter. All the measurements were performed at $28^{\circ} \mathrm{C}$, where neat DOPC and POPC vesicles are in the fluid phase, while DPPC and DSPC ones are in the gel phase. In fluid-state membranes, lipid molecules can diffuse freely within the bilayer plane, while in gel- state membranes lipids are more tightly packed and their motion is more constrained. As a consequence, gel- phase bilayers are expected to be stiffer than fluid- phase ones. Our results from the Force Spectroscopy FS analysis (Figure 1c, main text) confirms this behavior, with DPPC and DSPC vesicles being substantially stiffer than DOPC and POPC ones. Two other important parameters that can affect the stiffness of a lipid bilayer are the chain length and its degree of saturation; e. g. DSPC possesses two fully saturated chains, longer than all the other measured ones, resulting in the highest measured stiffness. Overall, the obtained stiffness values for neat DOPC, POPC, DPPC and DSPC vesicles (Figure 1c, main text) are in good agreement with results from recent AFM-FS investigations on similar vesicles ${ }^{10}$. Another interesting aspect to highlight is that the stiffnesses measured for the hybrid lipid vesicles (POPC/DPPC and DPPC/DSPC) have intermediate values with respect to liposomes made of the two pure components.

\section{AFM-based characterization of EVs size distribution and concentration}

AFM imaging was employed to obtain the size distribution of the EVs sample. A total of 179 EVs were imaged; from the topography of the AFM images, assuming the vesicle surface area conservation and by applying simple geometric consideration (see Ridolfi et al. ${ }^{11}$ for further details) it is possible to obtain the 
values of the diameter that the vesicles would have had in solution, prior to their adsorption to the surface (we refer to this parameter as "Size"). Figure S4 displays the size distribution for the EVs sample used in this study. The measured EVs have a mean size of $74 \mathrm{~nm}$ with a standard deviation of $30 \mathrm{~nm}$.

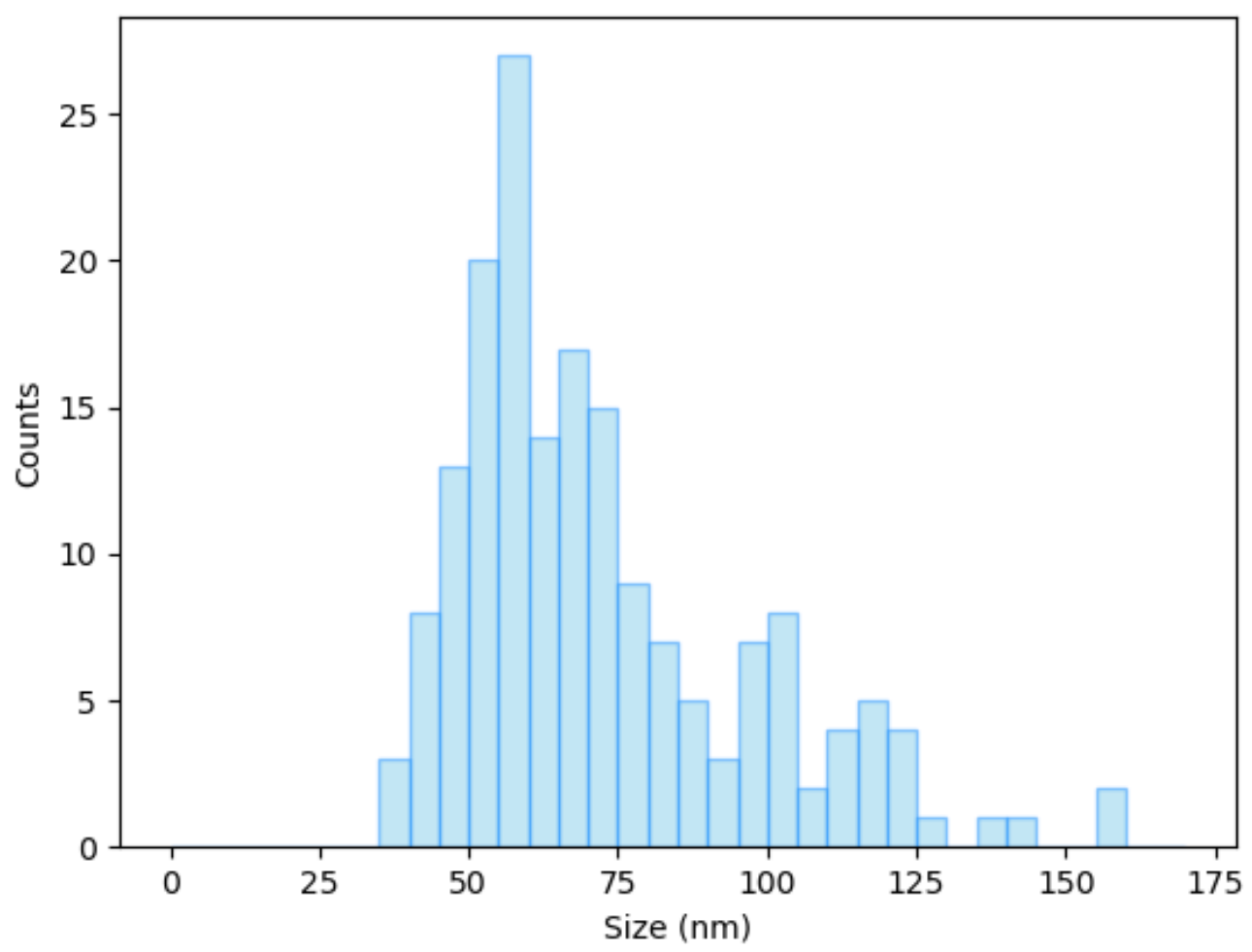

Figure S4: EVs size distribution obtained from the AFM imaging analysis. The size of the EVs (reported in the horizontal axis), indicates the diameter that the vesicles would have had in solution, prior to their adsorption.

AFM imaging was also used to estimate the starting concentration of the EVs sample. To do this, we compared the number of DPPC liposomes (coming from a solution with a known concentration and having a size distribution similar to the EVs) adsorbed on the glass surface with the number of EVs adsorbed on the same glass surface. This represents only a qualitative procedure and it is based on different assumptions: i) the interactions of DPPC liposomes with the glass surface are similar to the EVs ones, ii) the recorded images are representative of both the vesicles samples, iii) the size distributions of the two samples are similar to each other. The concentration of the DPPC starting solution is $0.02 \mathrm{mg} / \mathrm{ml}$; analysing the AFM images, we recorded a total of 329 vesicles in 4 different images, giving an average of 82.25 vesicles per image. Measuring EVs from TRAMP cells, we sampled 5 images, obtaining a total number of $166 \mathrm{EVs} ; 33.20 \mathrm{EVs}$ per image. From proportionality considerations, it is possible to estimate the concentration of the EVs, spotted on the glass coverslips, using the following expression:

DPPC concentration $(\mathrm{mg} / \mathrm{ml})$ :DPPC liposomes per image $=E V s$ concentration $(\mathrm{mg} / \mathrm{ml}):$ EVs per image From the expression we obtained a concentration of $0.008 \mathrm{mg} / \mathrm{ml}$ for the EVs sample. Since the EVs starting solution have been diluted six times before being spotted on the glass surface, the starting concentration is $0.048 \mathrm{mg} / \mathrm{ml}$. 


\section{Supplementary Characterization of liposomes/AuNPs hybrids}

\section{Small-Angle X-Ray Scattering}

SAXS measurements on liposomes/AuNPs hybrids were recorded at ID02 beamline, ESRF (Grenoble, France), using a sample-to-detector distance of $10 \mathrm{~m}$. The analysis of SAXS curves was carried out using Igor Pro ${ }^{3}$. SAXS measurements on liposomes/AuNPs aqueous dispersion were carried out in sealed glass capillaries of $1 \mathrm{~mm}$ diameter.

The SAXS profiles of DOPC liposomes/AuNPs and POPC liposomes/AuNPs in Figure $2 \mathrm{~b}$ were fitted according to a linear fit in the $0.0695-0.1142 \mathrm{~nm}^{-1} \mathrm{q}$-range, to obtain the slope values reported in the main text $(-1.5404 \pm 0.00297$ for DOPC and $-1.4987 \pm 0.00612$ for POPC). The fitting yielded a chisquare of 0.000239052 and 0.00106975 , for DOPC/AuNPs and POPC/AuNPs respectively.

The SAXS results of inset of Figure $2 b$ were collected at ID02 beamline, ESRF (Grenoble, France), using a sample-to-detector distance of $1 \mathrm{~m}$.

The scattering intensity $(I(q))$ is defined by the following equation:

$$
I(q)=K N_{p} V_{p}^{2}(\Delta \rho)^{2} P(q) S(q)+B
$$

With $\mathrm{k}$ instrumental constant, $\mathrm{N}_{\mathrm{p}}$ scattering nanoparticles' number per unit volume, $\mathrm{V}_{\mathrm{p}}$ nanoparticle's volume, $\Delta \rho$ contrast of the experiment, B background intensity, $\mathrm{P}(\mathrm{q})$ e $\mathrm{S}(\mathrm{q})$ form and structure factors, respectively.

In order to obtain the structure factor of the liposome/AuNPs complex, we divided the scattering intensity of the liposomes/AuNPs hybrid by the scattering intensity of the neat AuNPs dispersion (at a suitable dilution of $1: 10)$ :

$$
\frac{I(q)_{H y b}}{I(q)_{N P}} \sim \frac{S(q)_{H y b} P(q)_{H y b}}{S(q)_{N P} P(q)_{N P}}
$$

For a diluted AuNPs dispersion the structure factor can be considered equal to 1 . In addition, in the highq region (0.1-1.6 nm-1), the form factor of liposomes/AuNP hybrids can be approximated to the one of neat AuNPs, leading to the following:

$$
\frac{I(q)_{H y b}}{I(q)_{N P}}=S(q)_{H y b}
$$


The mean interparticle distance between the AuNPs within the aggregates (d) can be obtained from the $\mathrm{S}(\mathrm{q})$ vs $\mathrm{q}\left(\mathrm{nm}^{-1}\right)$ plot (see inset of Figure $2 \mathrm{~b}$ of the main text), by the following equation:

$$
d=\frac{2 \pi}{q \max }
$$

With $\mathrm{q}_{\max } \mathrm{q}$ value corresponding to the maximum of the correlation peaks reported in the inset of figure $2 b$ (main text).

\section{UV-Vis Spectroscopy}

The S.I. mean values for each liposomes/AuNPs mixtures are reported in Table S5, together with the relative standard deviation obtained from five repeated measurements on different samples (see "Preparation of liposomes/AuNPs hybrids" of SI).

\begin{tabular}{ll}
\hline & S.I. \\
\hline DOPC & $1.456 \pm 0.002$ \\
POPC & $1.438 \pm 0.001$ \\
POPC/DPPC & $1.377 \pm 0.005$ \\
DPPC & $1.16 \pm 0.01$ \\
DPPC/DSPC & $1.127 \pm 0.003$ \\
DSPC & $1.026 \pm 0.006$ \\
\hline
\end{tabular}

Table S5 S.I. mean value and standard deviation for each liposomes/AuNPs hybrid.

The fitting parameters describing the sigmoidal best fit (eqn. 1 of the main text) for the S.I. values of liposomes plotted versus the AFM-determined stiffness, reported in Figure $3 \mathrm{~b}$ of the main text, are the following:

\begin{tabular}{|c|c|c|c|c|}
\hline $\mathbf{a}$ & $\mathbf{b}$ & $\mathbf{c}$ & $\mathbf{d}$ & Chi square \\
\hline $1.4831 \pm$ & $-0.51151 \pm$ & $0.026043 \pm$ & $0.0063004 \pm$ & 0.0015 \\
0.0485 & 0.131 & 0.00266 & 0.00265 & \\
\hline
\end{tabular}

Table S6 Fitting parameters obtained by fitting the S.I. vs stiffness values of Figure 3c (main text) through the sigmoidal best fit (refer eqn 1 of main text for description of parameters).

The extent of AuNPs aggregation was also evaluated using different optical indexes, both taken from literature and defined in our lab. 
In particular, as an alternative to the bending index defined in the main text, which is based on the determination of the area under the absorbance curve associated to AuNP aggregation, we defined another optical parameter (S.I. (2)). This alternative bending index allows evaluating AuNPs aggregation extent by calculating the intensity difference between the free AuNPs primary plasmon band (at $521 \mathrm{~nm}$ ) and the aggregated AuNPs secondary plasmon peak, whose maximum is located at about $625 \mathrm{~nm}$ (see Figure S4). This result is then divided by the wavelength interval $(\Delta \lambda)$ between the two peaks and normalized for the S.I. of neat AuNPs.

$$
\operatorname{S.I.}(2)=\frac{I_{625}-I_{521}}{\Delta \lambda}
$$

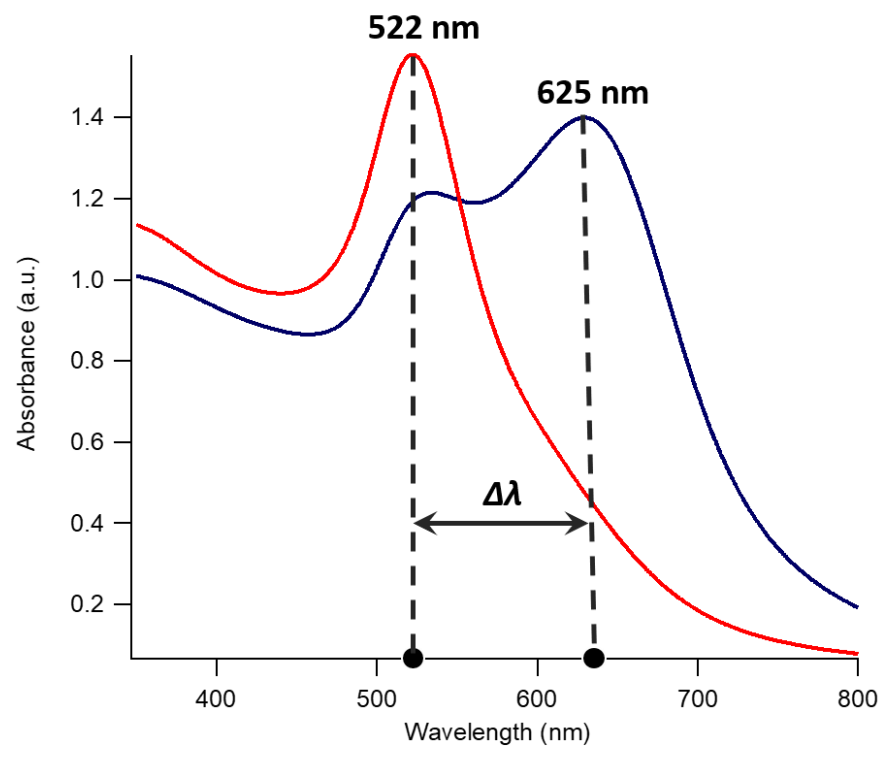

Figure S4 Visual description of the S.I. (2) evaluation.

We also selected another optical index from literature $\left(A . I_{\cdot C O N A N}\right)^{11,12}$, which is commonly used to describe the aggregation of AuNPs on natural and synthetic vesicles and defined as follows:

$$
A \cdot I \cdot \operatorname{CONAN}=\frac{I_{521}}{I_{650}+I_{800}}
$$

with $I_{521}, I_{650}$ and $I_{800}$ UV-Vis absorbances at 521,650 and $800 \mathrm{~nm}$ respectively.

Both the S.I. (2) and the A.I.CONAN show a sigmoidal behaviour as a function of membrane stiffness, as reported in Figure S5 and Table S7.

\begin{tabular}{lll}
\hline & S.I. (2) & A.I. CONAN \\
\hline DOPC & $-0.1965 \pm 0.0004$ & $0.785 \pm 0.014$ \\
POPC & $-0.14 \pm 0.03$ & $0.83 \pm 0.17$ \\
\hline
\end{tabular}




\begin{tabular}{lll}
\hline POPC/DPPC & $0.12 \pm 0.03$ & $1.02 \pm 0.05$ \\
DPPC & $0.71 \pm 0.06$ & $1.99 \pm 0.24$ \\
DPPC/DSPC & $0.76 \pm 0.01$ & $2.36 \pm 0.05$ \\
DSPC & $1.04 \pm 0.02$ & $3.28 \pm 0.24$
\end{tabular}

Table S7 S.I. (2) and A.I. conAN mean values and standard deviations for each liposomes/AuNPs hybrid.

\begin{tabular}{|c|c|c|c|c|}
\hline a & $-0.30138 \pm 0.189$ & & a & $0.5762 \pm 0.392$ \\
\hline b & $1.4174 \pm 0.387$ & Index (S. I. (2) or A.I. CONAN & b & $6.8394 \pm 12.2$ \\
\hline c & $0.023456 \pm 0.00288$ & $=a+\int \frac{b}{(c-\text { Stiffness }))}$ & c & $0.042282 \pm 0.0296$ \\
\hline d & $0.0062381 \pm 0.00288$ & $\left(\left(1+\exp \left(\frac{\mathrm{d}}{\mathrm{d}}\right)\right)\right)$ & d & $0.0096876 \pm 0.00795$ \\
\hline chi square & 0.018 & & chi square & 0.042 \\
\hline
\end{tabular}
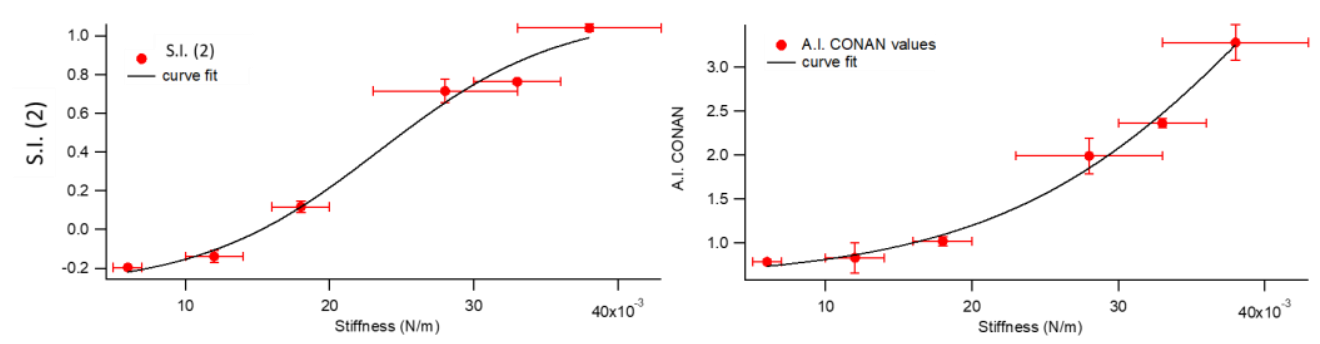

Figure S5 S.I. (2) and A.I. conan mean values and as a function of membrane stiffness. The sigmoidal fit curve is shown in black, together with the corresponding equation and fitting parameters (top inset). 